7-10-1998

\title{
Algebraic Shape Invariant Models
}

\author{
S Chaturvedi \\ University of Hyderabad \\ Ranabir Dutt \\ Visva Bharati University \\ Asim Gangopadhyaya \\ Loyola University Chicago, agangop@luc.edu \\ Prasanta K. Panigrahi \\ University of Hyderabad \\ C. Rasinariu \\ Columbia College Chicago, rasinariu@colum.edu
}

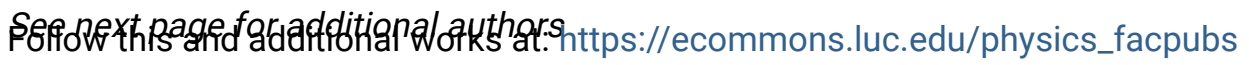

Part of the Quantum Physics Commons

\section{Author Manuscript}

This is a pre-publication author manuscript of the final, published article.

\section{Recommended Citation}

Chaturvedi, S; Dutt, Ranabir; Gangopadhyaya, Asim; Panigrahi, Prasanta K.; Rasinariu, C.; and Sukhatme, Uday P., "Algebraic Shape Invariant Models" (1998). Physics: Faculty Publications and Other Works. 34. https://ecommons.luc.edu/physics_facpubs/34

This Article is brought to you for free and open access by the Faculty Publications and Other Works by Department at Loyola eCommons. It has been accepted for inclusion in Physics: Faculty Publications and Other Works by an authorized administrator of Loyola eCommons. For more information, please contact ecommons@luc.edu. c) (†) $\Theta$

This work is licensed under a Creative Commons Attribution-Noncommercial-No Derivative Works 3.0 License. (C) 1998 The Authors. 
Authors

S Chaturvedi, Ranabir Dutt, Asim Gangopadhyaya, Prasanta K. Panigrahi, C. Rasinariu, and Uday P. Sukhatme 


\title{
Algebraic Shape Invariant Models
}

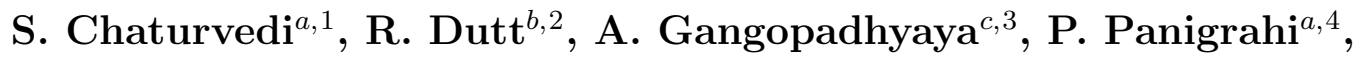 C. Rasinariu ${ }^{d, \text { p }}$ and U. Sukhatme ${ }^{d, \text { fo }}$}

\begin{abstract}
a) Department of Physics, University of Hyderabad, Hyderabad, India;
b) Department of Physics, Visva Bharati University, Santiniketan, India;

c) Department of Physics, Loyola University Chicago, Chicago, USA;

d) Department of Physics, University of Illinois at Chicago, Chicago, USA.
\end{abstract}

\begin{abstract}
Motivated by the shape invariance condition in supersymmetric quantum mechanics, we develop an algebraic framework for shape invariant Hamiltonians with a general change of parameters. This approach involves nonlinear generalizations of Lie algebras. Our work extends previous results showing the equivalence of shape invariant potentials involving translational change of parameters with standard $S O(2,1)$ potential algebra for Natanzon type potentials.
\end{abstract}

\footnotetext{
${ }^{1}$ scsp@uohyd.ernet.in

${ }^{2}$ rdutt@vbharat.ernet.in

3 agangop@luc.edu, asim@uic.edu

${ }^{4}$ panisp@uohyd.ernet.in

${ }^{5}$ costel@uic.edu

${ }^{6}$ sukhatme@uic.edu
} 
In recent years, there has been considerable interest in studying exactly solvable quantum mechanical problems using algebraic approaches[1-9]. In this respect, supersymmetric quantum mechanics (SUSYQM) has been found to be an elegant and useful prescription for obtaining closed analytic expressions both for the energy eigenvalues and eigenfunctions for a large class of one dimensional (or spherically symmetric three dimensional) problems [3]. The main ingredients in SUSYQM are the supersymmetric partner Hamiltonians $H_{-} \equiv A^{\dagger} A$ and $H_{+} \equiv A A^{\dagger}$. The $A$ and $A^{\dagger}$ operators used in this factorization are expressed in terms of the real superpotential $W$ as follows:

$$
A(x, a)=\frac{d}{d x}+W(x, a) \quad ; \quad A^{\dagger}(x, a)=-\frac{d}{d x}+W(x, a) .
$$

Here, $a$ is a parameter (or a set of parameters), which will play an important role in the approach of this paper. An interesting feature of SUSYQM is that for a shape invariant system [1-3], i.e. a system satisfying

$$
H_{+}\left(x, a_{0}\right)=H_{-}\left(x, a_{1}\right)+R\left(a_{0}\right) ; \quad a_{1}=f\left(a_{0}\right),
$$

the entire spectrum can be determined algebraically without ever referring to underlying differential equations.

Recently, it has been shown that shape invariant potentials in which the change of parameters $a_{1}=f\left(a_{0}\right)$ is of translational type $a_{1}=a_{0}+k, k=$ constant, possess a $S O(2,1)$ potential algebra and are solvable by group theoretical techniques[4-7]. Thus, for such potentials, there appear to be two seemingly independent algebraic methods, SUSYQM and potential algebras, for obtaining the complete spectra. One may naturally ask the question whether there is any connection between the two methods. Indeed, the equivalence between the two algebraic approaches has been established [6]. More specifically, for all shape invariant potentials of the Natanzon type [10], the underlying potential algebra is $S O(2,1)$. Other similar approaches are discussed in refs. [4, 11].

The purpose of this letter is to examine whether such an equivalence is also valid for a larger class of shape invariant potentials. We consider a much more general class of change of parameters, which contains both translations as well as scalings. In particular, for shape invariant potentials generated by a pure scaling ansatz [12] $a_{1}=q a_{0}, q=$ constant $(0<q<1)$, we find that the associated potential algebra is a nonlinear deformation of $S O(2,1)$. In fact, coordinate representations of these nonlinear algebras are an interesting area of research in their own right from a mathematical point of view [13]. We also show that the energy spectrum resulting from 
this potential algebra agrees with the results of SUSYQM.

To begin the construction of the operator algebra, let us express eq. (2) in terms of $A$ and $A^{\dagger}$ :

$$
A\left(x, a_{0}\right) A^{\dagger}\left(x, a_{0}\right)-A^{\dagger}\left(x, a_{1}\right) A\left(x, a_{1}\right)=R\left(a_{0}\right) .
$$

This relation resembles a commutator structure. To obtain a closed $S O(2,1)$ type Lie algebra, we introduce an auxiliary variable $\phi$ and define

$$
\begin{aligned}
& J_{+}=Q(\phi) A^{\dagger}\left(x, \chi\left(i \partial_{\phi}\right)\right), \\
& J_{-}=A\left(x, \chi\left(i \partial_{\phi}\right)\right) Q^{\star}(\phi),
\end{aligned}
$$

where $Q(\phi)$ is a function to be determined and $\chi$ is an arbitrary, real function. The operator $A\left(x, \chi\left(i \partial_{\phi}\right)\right)$ is given by eq. (11) with the substitution

$$
a \rightarrow \chi\left(i \partial_{\phi}\right)
$$

From eq. (州), one obtains

$$
\left[J_{+}, J_{-}\right]=Q(\phi) A^{\dagger}\left(x, \chi\left(i \partial_{\phi}\right)\right) A\left(x, \chi\left(i \partial_{\phi}\right)\right) Q^{\star}(\phi)-A\left(x, \chi\left(i \partial_{\phi}\right)\right) Q^{\star}(\phi) Q(\phi) A^{\dagger}\left(x, \chi\left(i \partial_{\phi}\right)\right)
$$

Choosing $Q(\phi)=e^{i p \phi}$, where $p$ is an arbitrary real constant, eq. (6) can be easily cast into the following form

$$
\left[J_{+}, J_{-}\right]=-\left\{A\left(x, \chi\left(i \partial_{\phi}\right)\right) A^{\dagger}\left(x, \chi\left(i \partial_{\phi}\right)\right)-A^{\dagger}\left(x, \chi\left(i \partial_{\phi}+p\right)\right) A\left(x, \chi\left(i \partial_{\phi}+p\right)\right)\right\} .
$$

In analogy with eq. (3), we impose the algebraic shape invariance condition

$$
A\left(x, \chi\left(i \partial_{\phi}\right)\right) A^{\dagger}\left(x, \chi\left(i \partial_{\phi}\right)\right)-A^{\dagger}\left(x, \chi\left(i \partial_{\phi}+p\right)\right) A\left(x, \chi\left(i \partial_{\phi}+p\right)\right)=R\left(\chi\left(i \partial_{\phi}\right)\right)
$$

Note that the quantity $J_{+} J_{-}$corresponds to the Hamiltonian

$$
H_{-}\left(x, i \partial_{\phi}+p\right)=A^{\dagger}\left(x, \chi\left(i \partial_{\phi}+p\right)\right) A\left(x, \chi\left(i \partial_{\phi}+p\right)\right)
$$

Identifying

$$
J_{3}=-\frac{i \partial_{\phi}}{p}
$$

we obtain a deformed $S O(2,1)$ Lie algebra whose generators $J_{+}, J_{-}$and $J_{3}$ satisfy

$$
\begin{aligned}
{\left[J_{3}, J_{ \pm}\right] } & = \pm J_{ \pm}, \\
{\left[J_{+}, J_{-}\right] } & =\xi\left(J_{3}\right)
\end{aligned}
$$


Here $\xi\left(J_{3}\right) \equiv-R\left(\chi\left(i \partial_{\phi}\right)\right)$. The algebraic shape invariance condition (3) is the main tool of our analysis. If we identify

$$
a_{0} \rightarrow \chi\left(i \partial_{\phi}\right) \quad ; \quad a_{1} \rightarrow \chi\left(i \partial_{\phi}+p\right)
$$

then depending on the choice of the $\chi$ function in eq. (12) we have:

1. translational models: $a_{1}=a_{0}+p$ for $\chi(z)=z$,

2. scaling models: $a_{1}=e^{p} a_{0} \equiv q a_{0}$ for $\chi(z)=e^{z}$.

One may note that in the translational reparametrization $(\chi(z)=z)$ the commutation relations (11) describe a standard, undeformed $S O(2,1)$ Lie algebra with $\xi\left(J_{3}\right)=-2 J_{3}$, as found in ref. [6]. Other changes of parameters follow from more complicated choices for $\chi(z)$. For example, if one takes $\chi(z)=e^{e^{z}}$, one gets the change of parameters $a_{1}=a_{0}^{2}$. Thus, with judicious choices for $\chi(z)$, various other reparametrizations can be emulated.

In this letter, we will analyze the potential algebra of scaling type reparametrizations. Different models with such multiplicative reparametrizations are characterized by the form of the remainder function $R\left(a_{0}\right)$. As a first example of scaling, we choose $R\left(a_{0}\right)=R_{1} a_{0}$. This choice generates self-similar potentials studied in refs. 12, 14. In this case, eqs. (11) become:

$$
\left[J_{3}, J_{ \pm}\right]= \pm J_{ \pm} \quad ; \quad\left[J_{+}, J_{-}\right]=\xi\left(J_{3}\right) \equiv-R_{1} \exp \left(-p J_{3}\right)
$$

Thus, we see that the resulting potential algebra is no longer given by a $S O(2,1)$ Lie algebra, but is deformed. To find the energy spectrum of the Hamiltonian $H_{-}$of eq. (9), we first construct the unitary representations of this deformed Lie algebra [15]. We define, up to an additive constant, a function $g\left(J_{3}\right)$ such that

$$
\xi\left(J_{3}\right)=g\left(J_{3}\right)-g\left(J_{3}-1\right)
$$

The Casimir of this algebra is then given $C_{2}=J_{-} J_{+}+g\left(J_{3}\right)$. In a basis in which $J_{3}$ and $C_{2}$ are diagonal, $J_{+}$and $J_{-}$play the role of raising and lowering operators, respectively. Operating on an eigenstate $|h\rangle$ of $J_{3}$, we have

$$
\begin{aligned}
J_{3}|h\rangle & =h|h\rangle, \\
J_{-}|h\rangle & =a(h)|h-1\rangle, \\
J_{+}|h\rangle & =a^{\star}(h+1)|h+1\rangle .
\end{aligned}
$$


Using eqs. (11) and (15) we obtain

$$
|a(h)|^{2}-|a(h+1)|^{2}=g(h)-g(h-1) .
$$

The profile of $g(h)$ determines the dimension of the unitary representation. To illustrate how this mechanism works, let us consider the two cases presented in fig. 1. One obtains finite

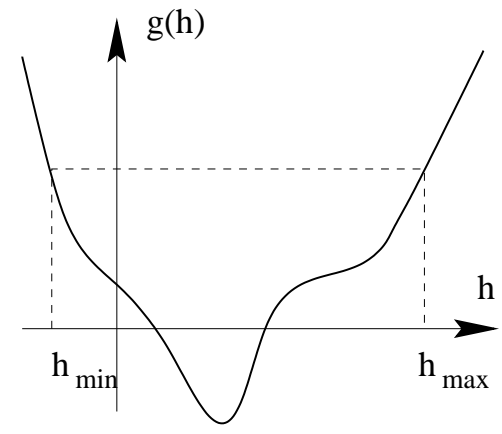

(a)

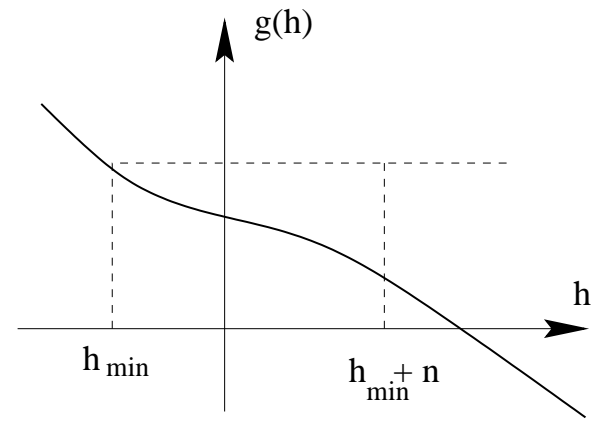

(b)

Figure 1: Generic behaviors of $g(h)$.

dimensional representations fig. 1a, by starting from a point on the $g(h)$ vs. $h$ graph corresponding to $h=h_{\min }$, and moving in integer steps parallel to the $h$-axis till the point corresponding to $h=h_{\max }$. Only those discrete values of $h_{\min }$ are allowed for which at the end points $a\left(h_{\min }\right)=$ $a\left(h_{\max }+1\right)=0$, and we get a finite representation. This is the case of $S U(2)$ for example, where $g(h)$ is given by the parabola $h(h+1)$. For a monotonically decreasing function $g(h)$, fig. $1 \mathrm{~b}$, there exists only one end point at $h=h_{\text {min }}$. Starting from $h_{\min }$ the value of $h$ can be increased in integer steps till infinity. In this case we have an infinite dimensional representation. As in the finite case, $h_{\min }$ labels the representation. However, the difference is that here $h_{\min }$ takes continuous values. Similar arguments apply for a monotonically increasing function $g(h)$. A more detailed discussion of these techniques is given in ref. [15].

In our case, from eqs. (13) and (14) one gets

$$
g(h)=\frac{R_{1}}{e^{p}-1} e^{-p h}=-\frac{R_{1}}{1-q} q^{-h} \quad ; \quad q=e^{p},
$$

represented graphically in fig. 2. Note that for scaling problems [12], one requires $0<q<$ 1 , which leads to $p<0$. From the monotonically decreasing profile of the function $g(h)$, it follows that the unitary representations of this algebra are infinite dimensional. If we label the lowest weight state of the operator $J_{3}$ by $h_{\min }$, then $a\left(h_{\min }\right)=0$. Without loss of generality 
we can choose the coefficients $a(h)$ to be real. Then one obtains from (16) for an arbitrary $h=h_{\min }+n, n=0,1,2, \ldots$

$$
a^{2}(h)=g(h-n-1)-g(h-1)=R_{1} \frac{q^{n}-1}{q-1} q^{1-h}
$$

where $q=e^{p}$. The spectrum of the Hamiltonian $H_{-}\left(x, a_{0}\right)$ is given by

$$
H_{-}|h\rangle=a^{2}(h)|h\rangle=R_{1} \frac{q^{n}-1}{q-1} q^{1-h}|h\rangle .
$$

Therefore, the eigenenergy is:

$$
E_{n}(h)=\alpha(h) \frac{q^{n}-1}{q-1} \quad ; \quad \alpha(h) \equiv R_{1} q^{1-h} .
$$

It is interesting to note that the eigenvalue $h$ of the operator $J_{3}$ plays the role of a parameter for the theory. To compare the spectrum of this Hamiltonian obtained in eq. (19) with those obtained from SUSYQM [12], we go to the $x$-representation. Here $|h\rangle \propto e^{i p h \phi} \psi_{h_{m i n}, n}(x)$ and hence, the Schrödinger equation for the Hamiltonian $H_{-}$reads

$$
\left\{-\frac{d^{2}}{d x^{2}}+W^{2}\left(x, e^{i \partial_{\phi}+p}\right)-W^{\prime}\left(x, e^{i \partial_{\phi}+p}\right)-E\right\} e^{i p \phi h} \psi_{h_{m i n}, n}(x)=0
$$

or

$$
\left\{-\frac{d^{2}}{d x^{2}}+W^{2}(x, \alpha)-W^{\prime}(x, \alpha)-E\right\} \psi_{h_{m i n}, n}(x)=0,
$$

which is exactly the Schrödinger equation for the scaling model in SUSYQM. Eigenenergies of this Hamiltonian [12] are indeed given by eq. (20). Hence, the energy $E$ is given by eq. (20) for $h$ $=$ constant. In fig. 2, we depict the elegant correspondence that exists between potential algebra and supersymmetric quantum mechanics for shape invariant potentials. Let us fix $h=h_{\min }+n$ to a given value, say $h_{0}$. For $n=0$ we have a representation of our deformed $\operatorname{SO}(2,1)$ algebra labeled by $h_{0} \equiv h_{\text {min }}$. We denote the corresponding potential from the Schrödinger equation of this Hamiltonian by $V_{0}$. From eq. (20) the energy $E_{0}\left(h_{0}\right)=0$ as we should expect for the ground state in SUSYQM. For $n=1$, we obtain the first excited state for $V_{0}$ at $E_{1}\left(h_{0}\right)=\alpha\left(h_{0}\right)$. Note that we can write $h_{0}=\left(h_{\text {min }}-1\right)+1 \equiv h_{1}+1$. Construct now a new representation labeled by $h_{1} \equiv h_{0}-1$. The corresponding potential $V_{1}$ has its ground state energy, at the same level with the first excited state of $V_{0}$. But this is the well known property of the supersymmetric partner potentials. One can now iterate this procedure. After $n$ steps, we build a representation labeled by $h_{n}$ where $h_{n}=h_{0}-n$, and the ground state energy of the corresponding potential $V_{n}$, will be exactly the $n$-excited state energy of $V_{0}$. 


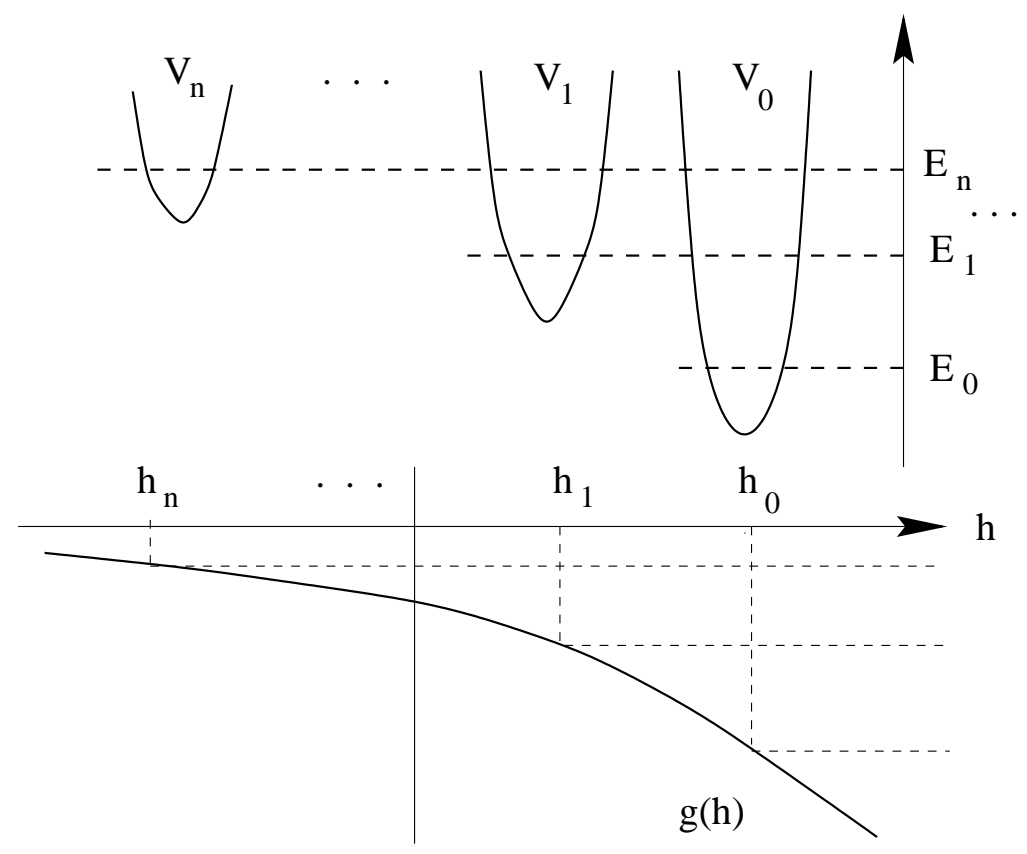

Figure 2: Schematic diagram showing the correspondence between potential algebra and SUSYQM with scaling type reparametrization.

For a more general case, we assume $R\left(a_{0}\right)=\sum_{j=1}^{\infty} R_{j} a_{0}^{j}$. In this case

$$
g(h)=\sum_{j=1}^{\infty} \frac{R_{j}}{1-e^{j p}} e^{-j p h}
$$

and therefore one gets

$$
\begin{aligned}
a^{2}(h) & =g(h-n-1)-g(h-1) \\
& =\sum_{j=1}^{\infty} \alpha_{j}(h) \frac{1-q^{j n}}{1-q^{j}}
\end{aligned}
$$

where $\alpha_{j}(h)=R_{j} e^{-j(h-1)}$. This result agrees with those obtained in ref. [12].

In conclusion, in this paper we have developed an algebraic approach involving nonlinear generalizations of Lie algebras for treating general shape invariant Hamiltonians.

A.G. acknowledges a research leave and a grant from Loyola University Chicago which made his involvement in this work possible. R.D. would also like to thank the Physics Department of the University of Illinois at Chicago for warm hospitality. Partial financial support from the U.S. Department of Energy is gratefully acknowledged. 


\section{References}

[1] L. Infeld and T.E. Hull, Rev. Mod. Phys. 23 (1951) 21.

[2] L. E. Gendenshtein, JETP Lett. 38 (1983) 356; L. E. Gendenshtein and I. V. Krive, Sov. Phys. Usp. 28 (1985) 645.

[3] F. Cooper, A. Khare, and U. Sukhatme, Phys. Rep. 251 (1995) 268 and references therein.

[4] Y. Alhassid, F. Gürsey and F. Iachello, Ann. Phys. 148 (1983) 346.

[5] J. Wu and Y. Alhassid, Phys. Rev. A31 (1990) 557; H. Y. Cheung, Nuovo Cimento B101 (1988) 193.

[6] A. Gangopadhyaya, J.V. Mallow and U.P. Sukhatme, Proceedings of Workshop on Supersymmetric Quantum Mechanics and Integrable Models, June 1997, Editor: H. Aratyn et al., Springer-Verlag.

[7] A. Gangopadhyaya, J.V. Mallow and U.P. Sukhatme, "Shape Invariant Natanzon Potentials from Potential Algebra"; to appear in Phys. Rev. A.

[8] A.B. Balantekin, Los Alamos e-print quant-ph/9712018.

[9] A. Barut, A. Inomata and R. Wilson, Jour. Phys. A20 (1987) 4075 and 4083.

[10] G. A. Natanzon, Teor. Mat. Fiz., 38 (1979) 219.

[11] P. Cordero and S. Salamo, Foundations of Physics 23 (1993) 675; Jour. Math. Phys. 35 (1994) 3301.

[12] D. Barclay, R. Dutt, A. Gangopadhyaya, A. Khare, A. Pagnamenta and U. Sukhatme, Phys. Rev. A48 (1993) 2786.

[13] J. Beckers, Y. Brihaye, and N. Debergh, Los Alamos preprint hep-th/9803253

[14] V.P. Spiridonov, Phys. Rev. Lett. 69 (1992) 298.

[15] M. Rocek, Phys. Lett. B255 (1991) 554. 